\title{
INYECTABLES \\ ANTIPSICÓTICOS DE LARGA DURACIÓN: Cuál y cuándo
}

Carlos Oliván Roldán Esteban Tercelán Álvarez Ma Pilar Sánchez Castillo Ángeles Fernández Sánchez

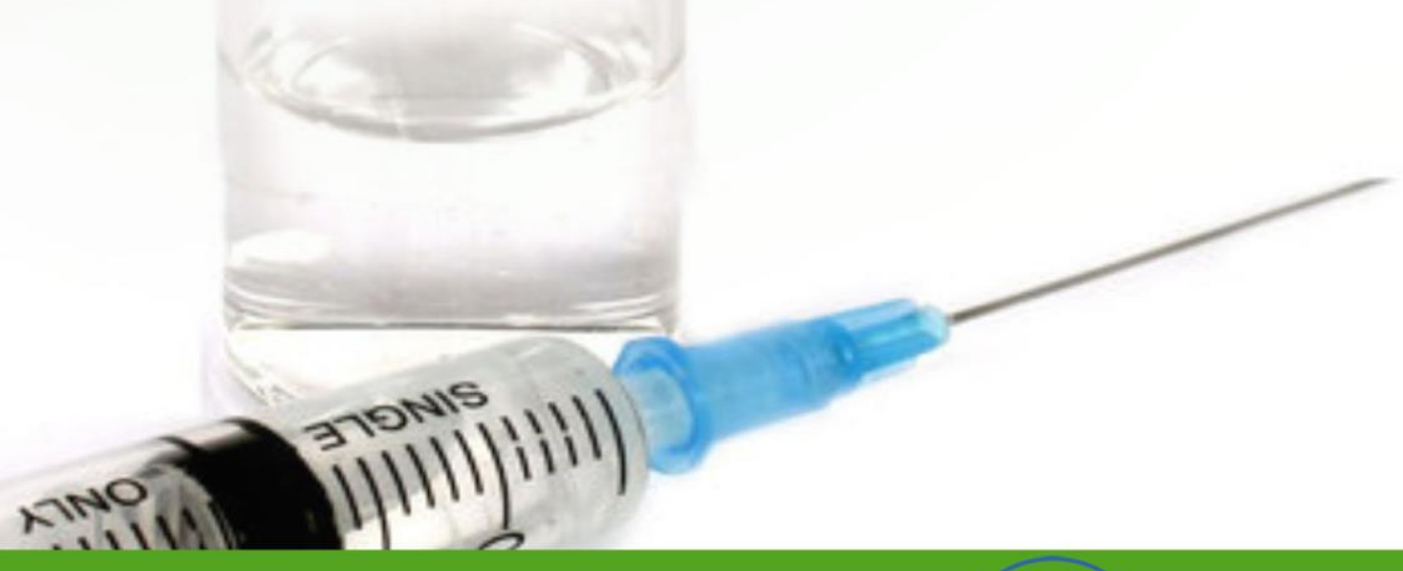




\section{INYECTABLES ANTIPSICOTICOS DE LARGA DURACION: Cuál y Cuándo}

\section{AUTORES}

Carlos Oliván Roldán

Psiquiatra, Responsable Clínico del Equipo de Tratamiento Asertivo Comunitario Área III, Servicio Murciano de Salud

Esteban Tercelán Álvarez Psiquiatra, Departamento 17 de Alicante, Servicio Valenciano de Salud (SERVASA)

Ma Pilar Sánchez Castillo

DUE Especialista en Salud Mental, Equipo de Tratamiento Asertivo Comunitario Área III, Servicio Murciano de Salud

Ángeles Fernández Sánchez DUE, Equipo de Tratamiento Asertivo Comunitario Área III, Servicio Murciano de Salud. Profesora Asociada Universidad de Murcia 


\section{${ }^{3}$ ciencias}

Editorial Área de Innovación y Desarrollo, S.L

Quedan todos los derechos reservados. Esta publicación no puede ser reproducida, distribuida, comunicada públicamente o utilizada, total o parcialmente, sin previa autorización.

(C) del texto: el autor

ÁREA DE INNOVACIÓN Y DESARROLLO, S.L.

C/ Els Alzamora, 17 - 03802 - ALCOY (ALICANTE) info@3ciencias.com

Primera edición: Julio 2016

ISBN: 978-84-945785-5-7

Registro: http://dx.doi.org/10.17993/Med.2016.22 
"Sólo se deberían escribir libros para decir cosas que uno no se atrevería a confiar a nadie"

\section{Emil Cioran}




\section{Contenido}

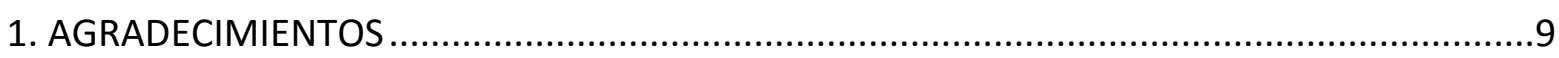

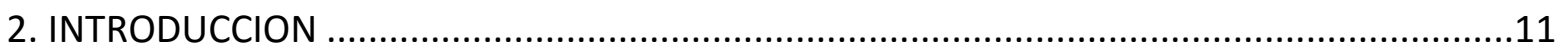

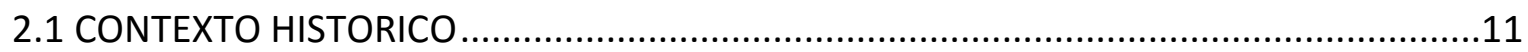

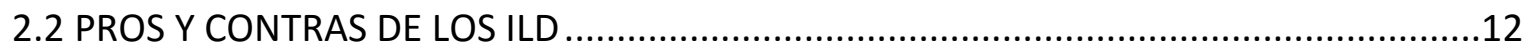

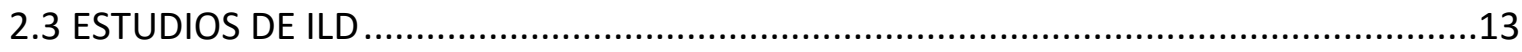

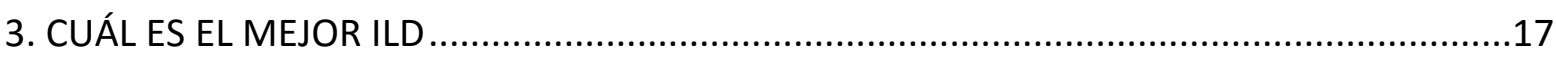

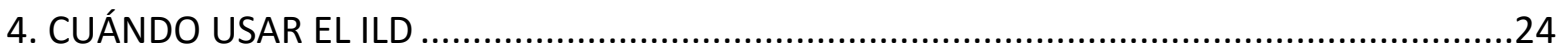

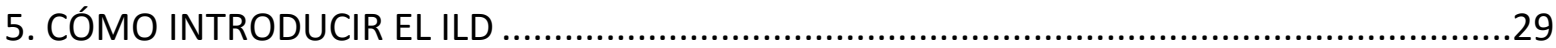

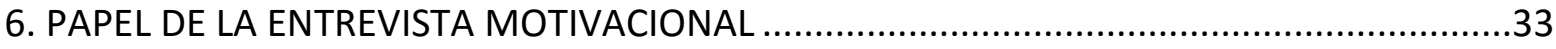

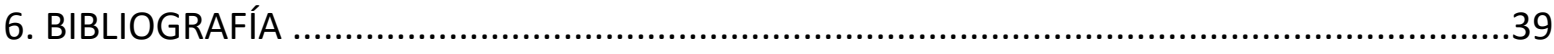




\section{AGRADECIMIENTOS}

El presente trabajo refleja parte del conocimiento y experiencia acumulados durante nuestro quehacer clínico diario: es por ello que en primer lugar el agradecimiento se dirige a aquellos pacientes y familiares que nos permiten de un modo u otro compartir sus vidas con nosotros.

En segundo lugar, la gratitud hacia la Asociación Astur Galaica de Tratamiento Asertivo Comunitario (ASGATAC) por permitirme colaborar con ellos como Miembro del Comité Científico de los Simposios Nacionales de Tratamiento Asertivo Comunitario que desde hace más de una década se celebran en la ciudad asturiana de Avilés, promoviendo un modelo integral de atención a las personas con Trastornos Mentales Graves basado en el respeto y la profesionalidad.

La filosofía que hay detrás de este libro "INYECTABLES ANTIPSICOTICOS DE LARGA DURACION: Cuál y Cuándo" es común a una ponencia oral presentada dentro de la decimo tercera edición del Simposio Nacional de Tratamiento Asertivo Comunitario en Salud Mental, avalado por la ASGATAC estrecha colaboración con la Asociación Asturiana de Rehabilitación Psicosocial (AARP)/FEARP. La excelente respuesta a la misma nos a animado a dejarla plasmada en un texto conciso que esperamos ofrezca perlas terapéuticas de interés para los clínicos interesados en la psicofarmacología.

Carlos Oliván Roldán

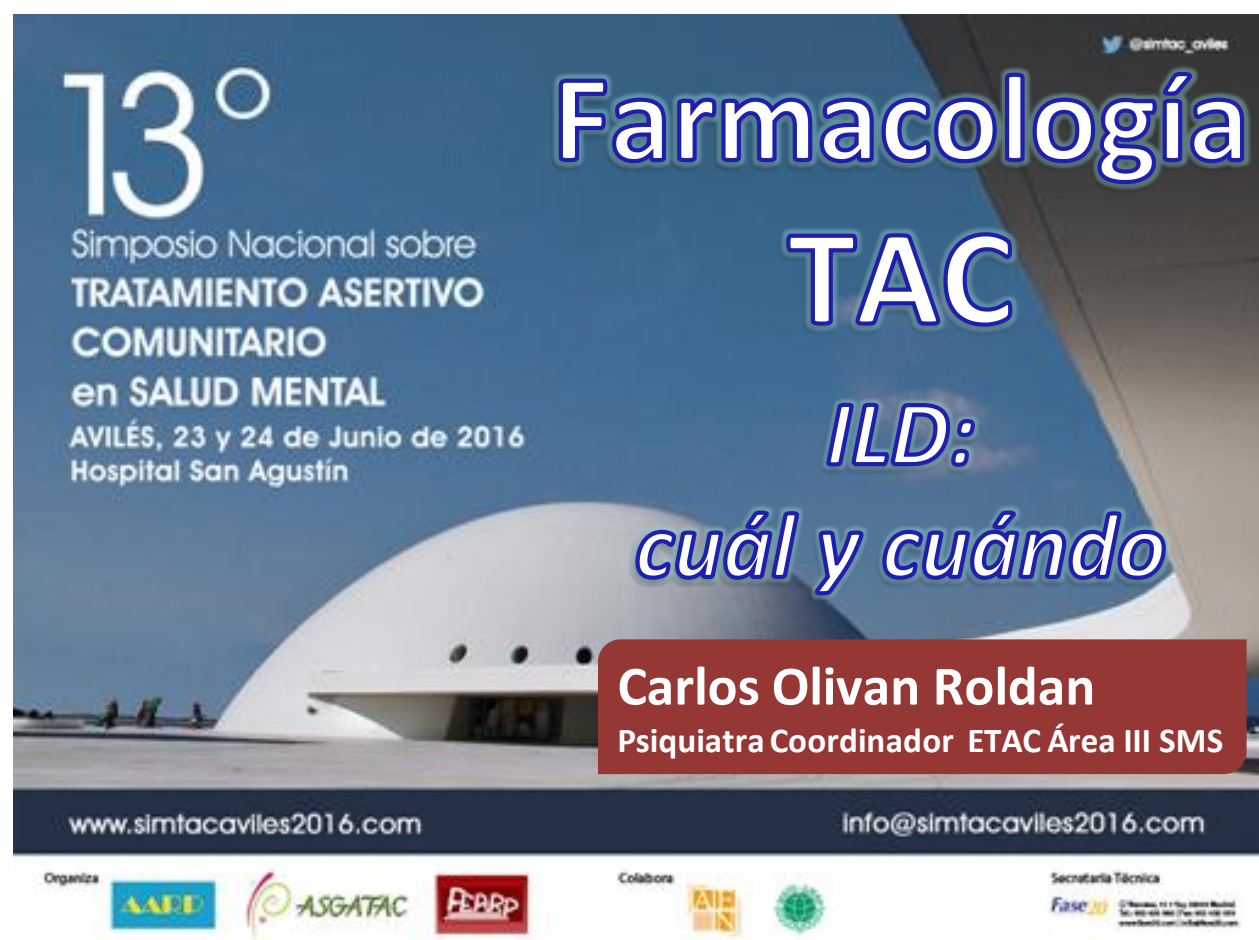

Figura 1: Simposio Nacional sobre tratamiento asertivo comunitario en salud mental. 


\section{INTRODUCCION}

El tema central de la elección del fármaco antipsicótico más adecuado para cada paciente determinado es una tarea harto compleja. Sin embargo, los clínicos suelen tener a menudo mayor pericia y menores reticencias a la hora de utilizar formulaciones orales en comparación con sus respectivas formulaciones inyectables de larga duración (ILD).

\section{La difícil decisión de elegir el mejor antipsicótico en el mejor momento para cada paciente}

Estos fármacos exigen quizá un mayor nivel de conocimientos psicofarmacológicos y no están exentos de particularidades especiales merecedoras de consideración.

En el momento de elaboración del presente trabajo, estos son los ILD disponibles en nuestro país:

\section{Antipsicóticos típicos}

\section{Antipsicóticos tipicos depot}

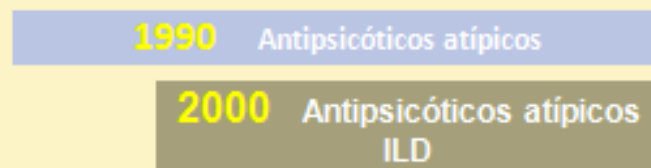

Modecate (decanoato de flufenazina)

*tenseren (éster palmítico de pipotiazina)

Clopixol depot (zuclopentixol decanoato)

*Haldol

(Haloperidol decanoato)

Risperdal Consta (risperidona inyectable acción prolongada)

Xeplion

(paliperidona inyectable acción prolongada)

Zypadhera

(olanzapina pamoato)

Maintena

(aripiprazol liberación prolongada)

Figura 2: ILD disponibles en España.

- El ILD de pipotiazina se retiró del mercado a partir de la Alerta Farmacéutica R15/2014.

- El ILD de haloperidol no está disponible de manera habitual pero puede obtenerse por los cauces previstos por la aemps para la solicitud de un medicamento no autorizado en España (medicación extranjera).

\subsection{CONTEXTO HISTORICO}

Quizás para poder entender mejor la situación actual, deberíamos realizar primero un breve repaso histórico de la aparición de los antipsicóticos. 
En la década de los 50, el mundo de la psiquiatra cambió radicalmente con la aparición de los primeros fármacos antipsicóticos de primera generación (APPG). Sin embargo, esta euforia inicial se enfrentó a la realidad de una pobre adherencia al tratamiento oral por parte de los pacientes.

En 1966, apareció el primer ILD: flufenazina enantato. Pasados unos 18 meses, apareció una variante del anterior que mejoraría la tolerabilidad del primero: ILD flufenazina decanoato. En 1981, hizo su aparición el ILD haloperidol decanoato.

Los investigadores de la época pudieron demostrar mediante ensayos clínicos aleatorizados una gran reducción de la morbilidad en la esquizofrenia gracias al empleo de estas formulaciones ILD.

Pueda quizá sorprender cual fue el impacto inicial que tuvieron estos ILD entre los clínicos de la época:

- Miedo a incremento de secundarismos

- Falta de eficacia

- Visión coercitiva de imposición del tratamiento sobre el paciente

En la década de los 80 y principios de los 90 hicieron su aparición los antipsicóticos de segunda generación (APSG), con las promesas de una mejor tolerancia y cierta capacidad de prevención de la neurotoxicidad asociada a los APPG.

De este modo, en la década de los 90 , se constató una reducción en el número de prescripciones de ILD clásicos. Sin embargo, en una repetición de la historia, los APSG tampoco pudieron solucionar los problemas de pobre adherencia terapéutica al tratamiento antipsicótico oral.

Finalmente, a partir del cambio de milenio, comenzó la comercialización de otro nuevo tipo de fármacos, con interesantes nuevas aportaciones y retos: los ILD de segunda generación o atípicos.

\subsection{PROS Y CONTRAS DE LOS ILD}

Existen si duda argumentos a favor y en contra para utilizar los ILD. En cada caso particular, y en función de cada clínico, pesarán más unos argumentos u otros. Pasamos a continuación a resumir los aspectos más importantes.

\section{Posibles argumentos a favor del uso de ILD:}

- No hay necesidad de administración diaria

- La administración queda garantizada y se logra transparencia de la adherencia

- Permite a los profesionales monitorizar e intervenir de manera apropiada si el paciente omite una dosis

- Existe menor probabilidad de síntomas de rebote y de recaídas abruptas

- Soluciona los problemas derivados de la adherencia parcial o no adherencia

- Si aparece una recaída, queda claro que obedece a motivos distintos a la no adherencia terapéutica

- Reduce el riesgo de sobredosis involuntaria o intencional

- Menor tasa de recaídas

- Mínimos problemas derivados de la absorción gastrointestinal

- Se logra una biodisponibilidad más consistente

- Existe en general una correlación más predecible entre dosis y niveles plasmáticos

- Se reducen los picos plasmáticos de fármaco 
- Se mejoran los resultados en el paciente

- Favorece un contacto regular entre el paciente y su equipo de salud mental

\section{Posibles argumentos en contra del uso de ILD:}

- Titulación de dosis lenta

- Precisa más tiempo para alcanzar niveles estables del fármaco

- Menor flexibilidad de ajuste de dosis

- Los efectos secundarios tardan más tiempo en desaparecer

- Dolor en el sitio de inyección

- Aparición de irritación y de lesiones en la zona de punción

- Su aplicación en la comunidad precisa de carga de tiempo de personal de enfermería, incluyendo la posibilidad de visitas domiciliarias

- ILD risperidona exige refrigeración

- Percepción de estigma

\subsection{ESTUDIOS DE ILD}

La esperanza inicial fue sin duda que, de manera global, los APSG fueran más eficaces que los APPG. Bajo esta premisa, ¿podrían descartarse los fármacos clásicos a la hora de elegir el mejor antipsicótico para un paciente?

Los estudios parecen querer decirnos que esta asunción inicial no era tan evidente:

Goff DC, Sullivan LM, McEvoy JP, et al. A comparison of ten-year cardiac risk estimates in schizophrenia patients from the CATIE study and matched controls. Schizophr Res2005;80:45-53.

Jones PB, Barnes TR, Davies L, et al. Randomized controlled trial of the effect on quality of life of second- vs first-generation antipsychotic drugs in schizophrenia: Cost Utility of the Latest Antipsychotic Drugs in Schizophrenia Study (CUtLASS 1). Arch Gen Psychiatry 2006;63:1079-87.

Leucht S, Corves C, Arbter D, et al. Second-generation versus first-generation antipsychotic drugs for schizophrenia: a meta-analysis. Lancet 2009;373:31-41. Rosenheck R, Lin H. Noninferiority of perphenazine vs. three second-generation antipsychotics in chronic schizophrenia. J Nerv Ment Dis 2014;202:18-24.

Rosenheck R, Perlick D, Bingham S, et al. Effectiveness and cost of olanzapine and haloperidol in the treatment of schizophrenia: a randomized controlled trial. IAMA 2003;290:2693-702.

Existen varios estudios como el CATIE, el CUtLASS o el famoso metaanálisis de Leucht en la revista Lancet nos sugieren que no hay evidencia consistente en la actualidad para sostener una menor eficacia global de los APPG sobre los APSG.

Además, no siempre es fácil encontrar evidencias científicas de fuentes fiables y es importante tener en cuenta la posibilidad de sesgos selectivos de publicación de resultados.

Hay que prestar especial atención en lo que se refiere a estudios psicofarmacológicos patrocinados por la industria farmacéutica, los cuales no tienen por qué moverse por los mismos intereses que los asociados a la investigación no privada.

A este respecto, siempre es interesante recordar el artículo de Heres et al. publicado en el American Journal of Psychiatry en Febrero de 2006, donde se analiza, tal y como anuncia el propio título del artículo: cuál es la explicación para que: 
- olanzapina sea mejor que risperidona

- risperidona sea mejor que quetiapina,

- quetiapina resulte finalmente mejor que olanzapina.

Por eso, si confiamos en que los metaanálisis pueden ofrecernos un grado máximo de evidencia científica, el artículo de Leucht publicado en Lancet en 2009 es una referencia básica.

En este artículo, se compara la eficacia y la tolerabilidad de 15 antipsicóticos distintos, encontrando que:

- En cuanto a eficacia: con la excepción del fármaco de elección para los cuadros de esquizofrenia refractaria a otros antipsicóticos, la clozapina, se demuestra claramente más eficaz que el resto de principios activos, existiendo todo un listado de distintos antipsicóticos que básicamente demuestran pequeñas diferencias de eficacia entre sí.

- En cuanto a tolerabilidad: los distintos fármacos antipsicóticos si presentan claros perfiles diferentes de efectos secundarios asociados

- Dada esta eficacia similar y los perfiles diversos de efectos secundarios, este autor, entre otros muchos, ha llegado a poner en tela de juicio la validez de la división de antipsicóticos entre viejos y nuevos, entre APPG y APSG.

Por lo menos, ¿contamos con evidencias para sostener que los ILD son mejores que los antipsicóticos orales?

En realidad, esta pregunta tampoco puede ser respondida de manera sencilla. Si revisamos estudios que enfrentan ILD con antipsicóticos orales, veremos que la respuesta de cuáles muestran resultados más favorables va a depender del propio diseño del estudio en cuestión.

\begin{tabular}{|c|c|c|c|c|c|}
\hline \multirow[t]{2}{*}{ Meta-Analy } & \multirow[b]{2}{*}{ Favors Depot } & \multirow[b]{2}{*}{ Favors Oral } & \multirow[b]{2}{*}{$\mathbf{R R}$} & \multirow[b]{2}{*}{$95 \% \mathrm{Cl}$} & \multirow[b]{2}{*}{ Weight } \\
\hline & & & & & \\
\hline RCTs & & & & & \\
\hline Gaebel et al, 201025 & $\longrightarrow$ & & 0.58 & $0.39-0.86$ & 15.13 \\
\hline Gaebel et al, $2010^{25}$ & 2 & & 0.50 & $0.38-0.67$ & 17.14 \\
\hline Kane et al, $2010^{26}$ & & - & 2.03 & $1.31-3.16$ & 14.38 \\
\hline Keks et at, $2007^{27}$ & - & - & 0.92 & $0.72-1.18$ & 17.77 \\
\hline Macfadden et al, $2010^{24}$ & & $=$ & 1.07 & $0.84-1.37$ & 17.76 \\
\hline Rosenheck et al, $2011^{7}$ & & - & 0.89 & $0.70-1.13$ & 17.82 \\
\hline Pooled RCTs & & - & 0.89 & $0.64-1.22$ & \\
\hline \multicolumn{6}{|l|}{ Prospective studies } \\
\hline Ciudad et al, $2008^{20}$ & & - & 0.80 & $0.60-1.06$ & 24.18 \\
\hline Kim et al, 2008 & & & 0.30 & $0.14-0.67$ & 8.15 \\
\hline Olivares et al, $2009^{5}$ & 0 & & 0.52 & $0.43-0.64$ & 28.07 \\
\hline Olivares et al, $2009^{\mathrm{s}}$ & & & 0.89 & $0.57-1.39$ & 17.07 \\
\hline Zhu et al, $2008^{29}$ & 皮 & & 0.59 & $0.43-0.81$ & 22.53 \\
\hline Pooled prospective studies & & & 0.62 & $0.48-0.81$ & \\
\hline \multicolumn{6}{|l|}{ Retrospective studies } \\
\hline Emsley et at, $2008^{30}$ & & & 0.25 & $0.09-0.70$ & 5.02 \\
\hline Emsley et al, $2008^{30}$ & & & 0.38 & $0.22-0.64$ & 15.06 \\
\hline Tavcar et al, $2000^{32}$ & & & 0.71 & $0.49-1.01$ & 26.45 \\
\hline Tiihonen et al, $2006^{31}$ & $\square$ & & 0.61 & $0.38-0.99$ & 17.91 \\
\hline Tihonen et al, $2011^{\mathrm{s}}$ & & & 0.16 & $0.02-1.05$ & 1.53 \\
\hline Tiihonen et al, $2011^{*}$ & $\square$ & & 0.69 & $0.35-1.35$ & 10.48 \\
\hline Tiihonen et al, $2011^{\mathrm{s}}$ & & & 0.46 & $0.16-1.34$ & 4.66 \\
\hline Twihonen et al, $2011^{\mathrm{s}}$ & & & 0.64 & $0.41-1.02$ & 18.89 \\
\hline Pooled retrospective studies & & & 0.56 & $0.44-0.71$ & \\
\hline
\end{tabular}

Figura 3: Estudios que enfrentan ILD con antipsicóticos orales. 
- Si analizamos estudios controlados aleatorizados a doble ciego, aparecen resultados contradictorios.

- Si por el contrario, analizamos estudios prospectivos, los resultados parecen favorecer discretamente a los ILD.

- Finalmente, si nos fijamos en estudios retrospectivos, aquellos que podrían ser considerados más cercanos a la práctica clínica real, la ventaja que ofrecen los ILD sobre los fármacos antipsicóticos orales parece aún más clara. 


\section{CuÁl ES EL MEJOR ILD}

Intentaremos en este apartado analizar algunos estudios que por sus resultados nos pueden resultar de especial relevancia, intentando en la medida de lo posible no incluir estudios patrocinados por la industria farmacéutica, que por otra parte, seguro ya serán conocidos por el lector por su lógica repercusión y presencia.

En esa línea, aún así mencionaremos a título exclusivamente informativo que existen varios artículos comparando ILD paliperidona frente a ILD risperidona o, más recientemente, ILD paliperidona frente a ILD aripiprazol.

Planteamos pues aquí otra serie de estudios, quizás menos conocidos, pero de igual o mayor relevancia sin duda alguna.

Pocos estudios han enfrentado ILD de primera con ILD de segunda generación.

Comenzaremos esta breve reseña de artículos con una comparación de ILD en población dual:

\section{Rubio G, Martinez I, Ponce G, et al. Long-acting injectable risperidone compared with zuclopenthixol in the treatment of schizophrenia with substance abuse comorbidity. Can J Psychiatry 2006;51:531-9.}

- Estudio de 6 meses de duración que incluye 115 pacientes con esquizofrenia y consumo de tóxicos.

- Los pacientes que recibieron ILD risperidona obtuvieron mejor resultado clínico en su proceso psicótico (medido por una mejoría de puntuación en la escala PANSS) respecto a aquellos pacientes que recibieron ILD zuclopentixol.

- Los pacientes que recibieron ILD risperidona también obtuvieron mejorías en su proceso adictivo (medido a través de una menor tasa de test positivos de orina para tóxicos y una mejor adherencia al programa psicoterapéutico) respecto a aquellos pacientes que recibieron ILD zuclopentixol.

\section{Lammers L, Zehm B, Williams R. Risperidone long-acting injection in schizophrenia spectrum illnesses compared to first generation depot antipsychotics in an outpatient setting in Canada. BMC Psychiatry 2013;13:155.}

- Pacientes con diagnóstico de esquizofrenia tratados con ILD risperidona enfrentado con pacientes que recibieron ILD de primera generación (ILD zuclopentixol, ILD flufenazina, ILD haloperidol, ILD pipotiazina...)

- Los resultados más Ilamativos fueron no encontrar diferencias significativas entre variables como discontinuación del tratamiento y hospitalización.

- Sin embargo, reforzando la idea esbozada previamente, los ILD de primera generación presentaron mayor incidencia que la ILD risperidona en la tasa de efectos secundarios debida a sintomatología extrapiramidal. 


\section{Covell NH, McEvoy JP, Schooler NR, et al. Effectiveness of switching from long-acting injectable fluphenazine or haloperidol decanoate to long-acting injectable risperidone microspheres: an open-label, randomized controlled trial. J Clin Psychiatry 2012;73:669-75.}

- Este estudio incluyó pacientes con esquizofrenia tratados con ILD haloperidol o ILD flufenazina.

- Los pacientes fueron aleatorizados bien a seguir con el mismo ILD clásico que previamente ya tenían prescrito o bien cambiar a un ILD de nueva generación, en concreto ILD risperidona.

- Los pacientes que cambiaron a ILD risperidona presentaron mayor tasa de discontinuación del tratamiento y mayores incrementos en variables metabólicas como el peso y niveles de la hormona prolactina.

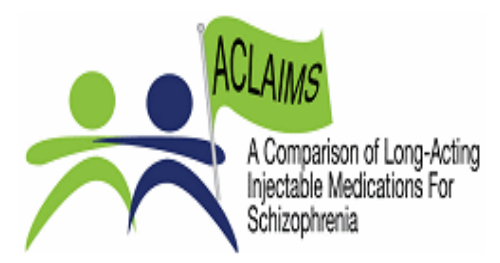

\section{A Comparison of Long-Acting Injectable Medications for Schizophrenia}

The ACLAIMS study is a multi-center randomized controlled trial of patients with schizophrenia or schizoaffective disorder who are expected to benefit from a long-acting injectable antipsychotic medication.

The study will compare the therapeutic effectiveness, costeffectiveness, tolerability (incidence and severity of adverse events), and enduring acceptability of two long-acting injectable antipsychotic preparations, paliperidone palmitate (PP) and haloperidol decanoate (HD), over a 2 year period of treatment and follow-up.

\footnotetext{
ACLAlliS is funded by the National Institute of Mental Health (NIIMH),

and is coordinated by the Schizophrenia Trials Network (STN), based at Columbia University, and collaborating with Duke University and the University of Texas Southwestern.
}

Figura 4: Comparación de la acción prolongada de medicamentos inyectables para la esquizofrenia.

- El estudio ACLAIMS es de especial importancia. Se trató de un estudio multicéntrico ( 22 centros de USA), aleatorizado y controlado a doble ciego en pacientes con esquizofrenia y trastorno esquizoafectivo (un total de 311).

- Esta investigación comparó la eficacia, coste, tolerabilidad y aceptabilidad de dos ILD: ILD palmitato de paliperidona e ILD haloperidol. El periodo de observación del estudio fue de 24 meses, lo cual añade un extra de valor a la hora de interpretar los resultados hallados en el artículo, cuya cita es: 
McEvoy JP, Byerly M, Hamer RM, et al. Effectiveness of paliperidone palmitate vs haloperidol decanoate for maintenance treatment of schizophrenia: a randomized clinical trial. JAMA 2014;311:1978-87.

- El artículo fue publicado en JAMA en 2014. Los resultados principales fueron que la ratio de fracaso por falta de eficacia no fue significativamente distinta entre ambos grupos: Un 33.8\% de los pacientes que recibieron ILD palmitato de paliperidona presentaron un fracaso por ineficacia del fármaco, mientras que el porcentaje de fracasos en el grupo de pacientes que recibieron ILD haloperidol ascendió al $32.4 \%$.

- Respecto al perfil de efectos secundarios, sí aparecieron diferencias significativas entre ambos grupos. Los pacientes asignados al grupo de ILD palmitato de paliperidona ganaron más peso, mientras que los que recibieron ILD haloperidol lo perdieron. Los pacientes que recibieron ILD paliperidona presentaron de manera significativa mayores niveles medios de prolactina sérica. Los pacientes asignados al grupo de ILD haloperidol tuvieron significativamente mayores tasas globales de acatisia.

- Este estudio, dado su diseño, no despeja la duda del efecto neurotóxico comparativo entre ambos grupos a más largo plazo, aunque no parece ilógico presuponer con los datos actuales de evidencia, que el efecto neurotóxico sería mayor para el ILD haloperidol.

JOURNAL OF

MARKET ACCESS \&

HEALTH POLICY

EFFICACY, TOLERABILITY, AND SAFETY OF ARIPIPRAZOLE ONCEMONTHLY VERSUS OTHER LONG-ACTING INJECTABLE ANTIPSYCHOTIC THERAPIES IN THE MAINTENANCE TREATMENT OF SCHIZOPHRENIA: A MIXED TREATMENT COMPARISON OF DOUBLEBLIND RANDOMIZED CLINICAL TRIALS

Istvan M. Majer, PhD ${ }^{1^{*}}$, Fiona Gaughran, MD $\stackrel{2.3}{ }$, Christophe Sapin, MSc $\frac{4}{4}$, Maud Beillat, MSc $\underline{5}$ and Maarten Treur, MSc 1

- Otro estudio muy interesante y recientemente publicado (Septiembre de 2015) reúne una comparación de ensayos clínicos aleatorizados a doble ciego donde analiza la eficacia, tolerabilidad y seguridad del LAl aripiprazol respecto a varios ILD como son ILD risperidona, ILD palmitato de paliperidona, ILD pamoato de olanzapina e ILD haloperidol.

- Los análisis finales sugieren que todos estos ILD presentan una eficacia similar, redundando de nuevo en la idea que venimos presentando. 


\section{ClinicalTrials.gov}

A service of the U.S. National Institutes of Health

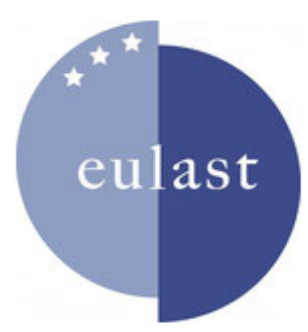

\section{European Long-acting Antipsychotics in Schizophrenia Trial (EULAST)}

This study is currently recruiting participants.

Verified February 2016 by UMC Utrecht

Sponsor:

UMC Utrecht

Information provided by (Responsible Party):

Rene Kahn, UMC Utrecht
ClinicalTrials.gov Identifier:

NCT02146547

First received: May 21, 2014

Last updated: February 5, 2016

Last verified: February 2016

History of Changes

- El estudio EULAST está reclutando pacientes en el momento de elaboración de este trabajo.

- Se trata de un estudio de fase 4, multicéntrico, multinacional europeo, con una duración de 18 meses de seguimiento para pacientes con esquizofrenia.

- Los pacientes van a ser aleatorizados a 4 comparadores activos: aripiprazol oral, ILD aripiprazol, paliperidona oral e ILD paliperidona.

- El estudio final pretende que la muestra estimada incluya unos 600 pacientes

- El final del estudio está previsto para el año 2018, momento en el que podremos obtener respuestas muy interesantes acerca de la comparación real entre un mismo principio activo prescrito por dos vías diferentes: oral e ILD.

\section{En resumen, no queda claro que los nuevos ILD sean más eficaces que los antiguos \\ Sí parecen existir claras diferencias entre los distintos ILD en cuanto a tolerabilidad}

Si parte de nuestra propuesta inicial era dilucidar cuál puede ser el mejor antipsicótico ILD disponible, queda claro que a día de hoy, no parece que contemos con un nivel de evidencia suficiente para poder recomendar uno sobre otro en función de su eficacia. 


\section{Los clínicos debemos elegir el mejor ILD para cada paciente en función de:}

\section{Las preferencias del paciente}

Llegados a este punto, merece la pena revisar brevemente el perfil de tolerabilidad descrito para los distintos antipsicóticos. La literatura, también en este punto, puede llegar a ser confusa e incluso con resultados contradictorios y aparentemente mutuamente excluyentes.

Resulta más relevante utilizar fuentes de información sin conflicto de intereses para obtener la evidencia científica de mayor solidez posible. Recomendamos revisar, por ejemplo, manuales como la Guía de Prescripción en Psiquiatria del Hospital Maudsley, en su reciente doceava edición.

Exponemos a continuación una tabla comparativa con el perfil de efectos secundarios más frecuentemente asociados a los diversos antipsicóticos:

- En amarillo quedan resaltado los principios activos de los que disponemos formulación ILD.

\begin{tabular}{|c|c|c|c|c|c|c|c|}
\hline Drug & Sedation & $\begin{array}{l}\text { Weight } \\
\text { gain }\end{array}$ & Akathisia & Parkinsonism & $\begin{array}{l}\text { Anti- } \\
\text { cholinergic }\end{array}$ & Hypotension & $\begin{array}{l}\text { Prolactin } \\
\text { elevation }\end{array}$ \\
\hline Amisulpride & - & + & + & + & - & - & +++ \\
\hline Aripiprazole & - & - & + & - & - & - & - \\
\hline Asenap ine & + & + & + & - & - & - & + \\
\hline Benperidol & + & + & + & +++ & + & + & +++ \\
\hline Chlorpromazine & +++ & ++ & + & ++ & ++ & ++ & ++ \\
\hline Clozapine & +++ & +++ & - & - & +++ & ++ & - \\
\hline Flupentixol & + & ++ & ++ & ++ & ++ & + & +++ \\
\hline Fluphenazine & + & + & ++ & ++ & ++ & + & +++ \\
\hline Haloperidol & + & + & +++ & ++ & + & + & ++ \\
\hline Iloperidone & - & ++ & + & + & - & + & - \\
\hline Loxapine & $H$ & + & + & ++ & + & ++ & ++ \\
\hline Lurasidone & + & - & + & + & - & - & + \\
\hline Olanzapine & ++ & +++ & + & - & + & + & + \\
\hline Paliperidone & + & ++ & + & + & + & ++ & ++ \\
\hline Perphenazine & + & + & ++ & +++ & + & + & ++ \\
\hline Pimozide & + & + & + & + & + & + & ++ \\
\hline Pipothi azine & ++ & ++ & + & ++ & ++ & ++ & +++ \\
\hline Promazine & +++ & ++ & + & + & ++ & ++ & ++ \\
\hline Quetiap ine & ++ & ++ & - & - & + & ++ & - \\
\hline Risperidone & + & ++ & + & + & + & ++ & +++ \\
\hline Sertindole & - & + & - & - & - & +++ & - \\
\hline Sulpiride & - & + & + & + & - & - & +++ \\
\hline Trifluoperazine & + & + & + & +++ & + & + & ++ \\
\hline Zprasidone & + & - & + & - & - & + & + \\
\hline Zuclopenthixol & ++ & ++ & + & ++ & ++ & + & +++ \\
\hline
\end{tabular}

Tabla 1: Perfil de efectos secundarios más frecuentemente asociados a los diversos antipsicóticos 
- El aripiprazol es una molécula con un perfil de escasos efectos adversos a nivel de: sedación, ganancia de peso, acatisia, parkinsonismo, hipotensión y elevación de la prolactina

- La flufenazina es una molécula con un perfil bajo de sedación, bajo de ganancia de peso, moderado de acatisia, elevado de parkinsonismo, moderado de efectos anticolinérgicos, bajo de hipotensión y elevado de elevación de prolactina.

- El haloperidol es una molécula con un perfil bajo de sedación, bajo de ganancia de peso, elevado de acatisia, elevado de parkinsonismo, bajo de efectos anticolinérgicos, bajo de hipotensión y moderado de elevación de prolactina.

- La olanzapina es una molécula con un perfil moderado de sedación, elevado de ganancia de peso, bajo de acatisia, muy bajo de parkinsonismo, bajo de efectos anticolinérgicos, bajo de hipotensión y bajo de elevación de prolactina.

- La paliperidona es una molécula con un perfil bajo de sedación, moderado de ganancia de peso, bajo de acatisia, bajo de parkinsonismo, bajo de efectos anticolinérgicos, moderado de hipotensión y elevado de elevación de prolactina.

- La risperidona es una molécula con un perfil bajo de sedación, moderado de ganancia de peso, bajo de acatisia, bajo de parkinsonismo, bajo de efectos anticolinérgicos, moderado de hipotensión y elevado de elevación de prolactina.

- El zuclopentixol es una molécula con un perfil moderado de sedación, moderado de ganancia de peso, moderado de acatisia, moderado de parkinsonismo, moderado de efectos anticolinérgicos, bajo de hipotensión y elevado de elevación de prolactina.

Muchas veces, en la decisión de la elección del ILD influirán los antecedentes de mala tolerancia que haya presentado el paciente en tratamientos previos con antipsicóticos.

La siguiente tabla refleja:

- En la primera columna: un listado de Efectos Secundarios provocados por el antipsicótico previo;

- En la segunda columna, los fármacos de elección propuestos para el cambio de antipsicótico;

- Por último, la tercera columna enumera los posibles cambios de fármacos como estrategias de segunda línea a adoptar tras un fracaso en la estrategia de primera línea.

De nuevo, en amarillo quedan resaltados los principios activos de los que disponemos formulación ILD. 


\begin{tabular}{|c|c|c|}
\hline Acute EPS & $\begin{array}{l}\text { Aripiprazole } \\
\text { Olanzapine } \\
\text { Quetiapine }\end{array}$ & $\begin{array}{l}\text { Clozapine } \\
\text { Lurasidone } \\
\text { Ziprasidone }\end{array}$ \\
\hline Dyslipidaemia & $\begin{array}{l}\text { Amisulpride } \\
\text { Aripiprazole } \\
\text { Lurasidone } \\
\text { Zpprasidone }\end{array}$ & Asenapine \\
\hline $\begin{array}{l}\text { Impaired glucose } \\
\text { tolerance }\end{array}$ & $\begin{array}{l}\text { Amisulpride } \\
\text { Aripiprazole } \\
\text { Lurasidone } \\
\text { Zprasidone }\end{array}$ & $\begin{array}{l}\text { Risperidone } \\
\text { Haloperidol }\end{array}$ \\
\hline Hyperprolactinaemia & $\begin{array}{l}\text { Aripiprazole } \\
\text { Lurasidone } \\
\text { Quetiapine }\end{array}$ & $\begin{array}{l}\text { Clozapine } \\
\text { Olanzapine } \\
\text { Ziprasidone }\end{array}$ \\
\hline Postural hypotension & $\begin{array}{l}\text { Amisulpride } \\
\text { Aripiprazole } \\
\text { Lurasidone }\end{array}$ & $\begin{array}{l}\text { Haloperidol } \\
\text { Sulpiride } \\
\text { Triflucperazine }\end{array}$ \\
\hline Sedation & $\begin{array}{l}\text { Amisulpride } \\
\text { Aripiprazole } \\
\text { Risperidone } \\
\text { Sulpiride }\end{array}$ & $\begin{array}{l}\text { Haloperidol } \\
\text { Trifuoperazine } \\
\text { Ziprasidone }\end{array}$ \\
\hline Sexual dysfuction & $\begin{array}{l}\text { Aripiprazole } \\
\text { Quetiapine }\end{array}$ & $\begin{array}{l}\text { Clozapine } \\
\text { Lurasidone }\end{array}$ \\
\hline Tardive dyskinesia & Clozapine & $\begin{array}{l}\text { Aripiprazole } \\
\text { Olanzapine } \\
\text { Quetiapine }\end{array}$ \\
\hline Weight gain & $\begin{array}{l}\text { Amisulpride } \\
\text { Aripiprazole } \\
\text { Haloperidd } \\
\text { Lurasidone } \\
\text { Zprasidone }\end{array}$ & $\begin{array}{l}\text { Asenapine } \\
\text { Trifluoperazine }\end{array}$ \\
\hline
\end{tabular}

Tabla 2: Efectos secundarios, fármacos y cambios de fármacos de forma estratégica.

- Ante sintomatología extrapiramidal aguda: aripiprazol y olanzapina son buenas opciones a tener en cuenta.

- Ante dislipemia: el aripiprazol es una buena elección.

- Ante problemas con el metabolismo de la glucosa: aripiprazol como primera línea, risperidona y haloperidol de segunda línea.

- Ante hiperprolactinemia: aripiprazol como primera línea, olanzapina de segunda línea.

- Ante hipotensión postural: aripiprazol como primera línea, haloperidol de segunda línea.

- Ante prolongación del intervalo QT: aripiprazol como primera línea así como paliperidona con monitorización electrocardiográfica.

- Ante sedación: aripiprazol y risperidona como primera línea, haloperidol de segunda línea.

- Ante disfunción sexual: aripiprazol como primera línea.

- Ante discinesia tardía: aripiprazol y olanzapina como opciones de segunda línea tras la clozapina, de la que no hay disponible formulación ILD.

- Ante aumento de peso: aripiprazol y haloperidol como primera línea. 


\section{Cuándo uSAR El iLd}

Uno de los debates siempre presentes en el uso de los ILD es elegir a partir de qué momento evolutivo dentro del curso de la esquizofrenia es pertinente utilizar estos fármacos.

Sin entrar en la polémica de tratar o no farmacológicamente a los pacientes en fases premórbidas o de riesgo, la incógnita se centra principalmente en si destinar los ILD a aquellos cuadros con deterioro establecido o en fase crónica residual, o si acaso es apropiado e incluso beneficioso abordar los casos desde las fases más iniciales, como lo sería el primer episodio psicótico.

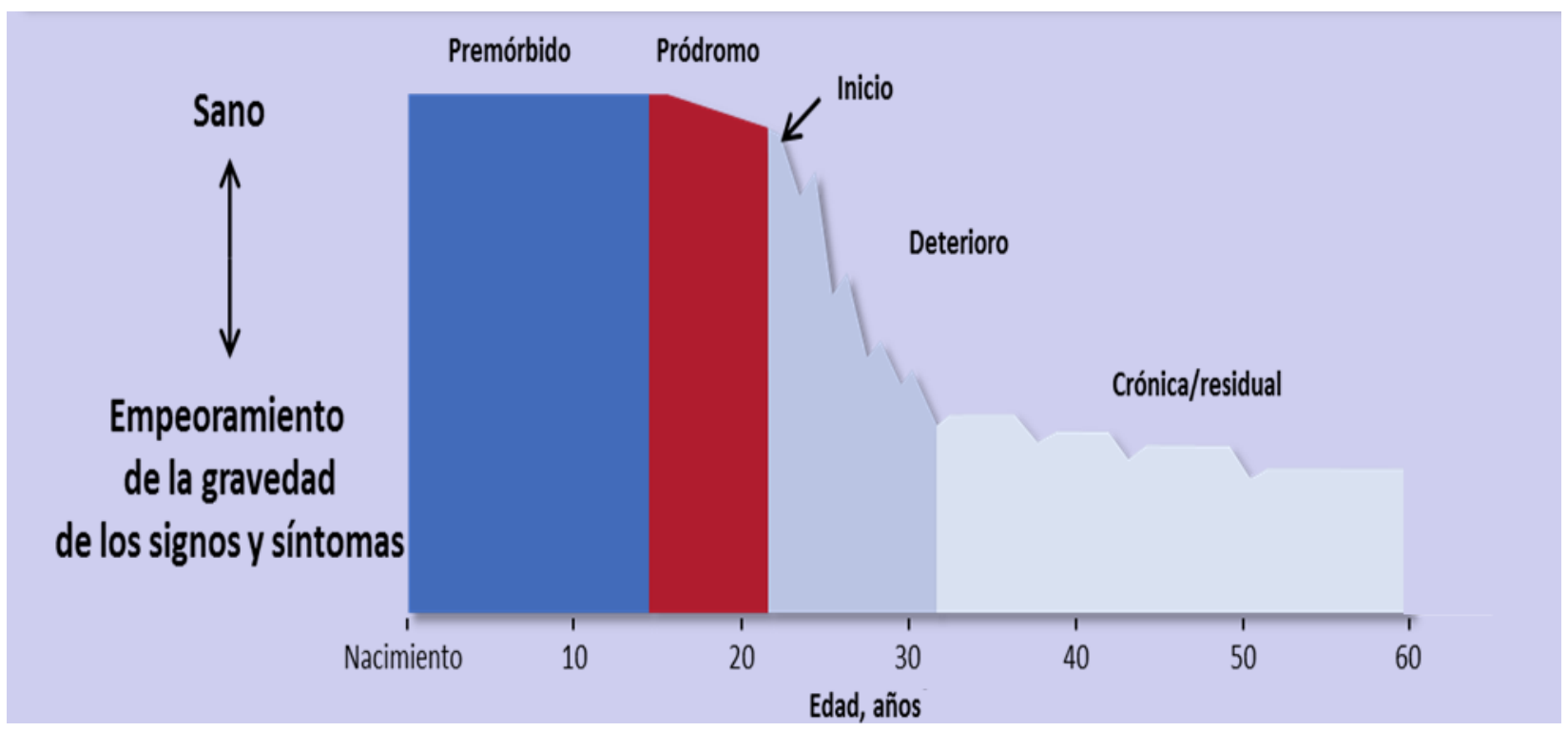

Gráfico 1: Evolución del empeoramiento de la gravedad de los síntomas por años.

Diversos artículos han abordado las actitudes de los profesionales de la salud mental acerca del uso de los ILD.

En el interesante artículo de Geerts et al, 891 psiquiatras y enfermeras europeas expresaron su opinión acerca del mejor momento dentro del curso evolutivo de la esquizofrenia para instaurar uno de estos fármacos. Un porcentaje del $96 \%$ prefirieron utilizar un ILD sobre el oral para aquellos casos de esquizofrenia crónica, pero sólo un $40 \%$ expresaron la misma preferencia para aquellos pacientes en su primer episodio psicótico.

En todo caso, hay una gran diversidad de investigaciones que no hacen sino confirmar que es constante la sobreestimación que los psiquiatras tienen sobre el cumplimiento terapéutico oral de sus pacientes.

\footnotetext{
Geerts, P., Martinez, G. and Schreiner, A. (2013) Attitudes towards the administration of long-acting antipsychotics: a survey of physicians and nurses. BMC Psychiatry 13: 58.
}

No hay que olvidar que las dificultades con la adherencia terapéutica son intrínsecas a todos aquellos procesos de salud crónicos, y que son múltiples las motivaciones que tienen los pacientes para no cumplir el tratamiento prescrito. 


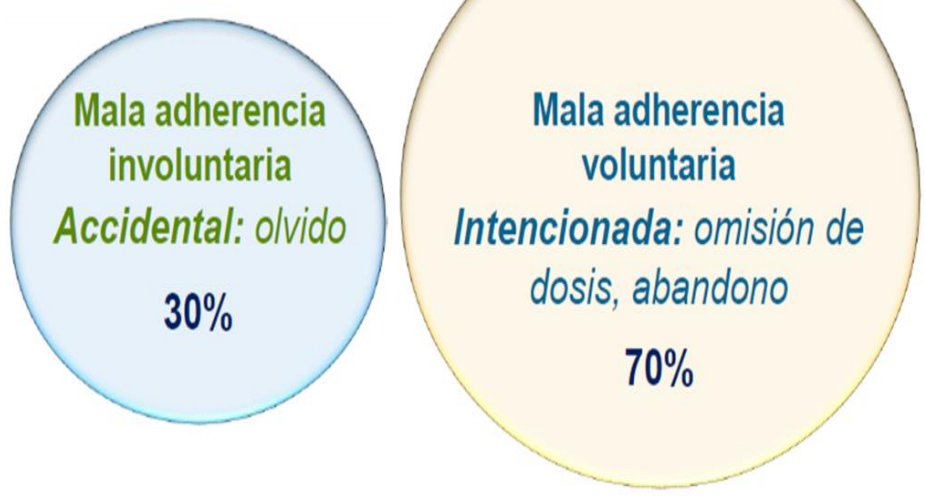

Figura 5: Porcentajes de la mala adherencia voluntaria e involuntaria.

A modo de ejemplo, la siguiente tabla recoge los diferentes grados de adherencia terapéutica al tratamiento farmacológico prescrito para distintas enfermedades.

\section{Medicamentos}

\section{Antibióticos}

(en procesos infecciosos no graves)

\section{Antineoplásicos orales}

\section{Antidepresivos}

\section{Antipsicóticos}

(en esquizofrenia)

\section{Inmunosupresores}

(en transplante renal)

\section{Antihipertensivos}

\section{Antirretrovirales}

\section{Hipolipemiantes}

\section{Grado de adherencia}

\section{$39 \%$ (recuento)}

$80 \%$ (conjunto de estudios con monitorización elect., recuento, autocomunicado y metabolitos)

$56-72 \%$ (recuento, autocomunicado)

$<50 \%$ (metabolitos)

$78 \%$ (autocomunicado, monitorización elect.)

$73 \%$ (una dosis diaria)

$55,5 \%$ (entrevista)

$46,5 \%$ (recuento)

\section{$56-83 \%$}

$64 \%$ (autocomunicado)

Tabla 3: Diferentes grados de adherencia terapéutica al tratamiento farmacológico prescrito.

En este contexto, parece lícito preguntarse si, a pesar de conocer las bajas tasas de adherencia oral al tratamiento, los pacientes con un primer episodio psicótico deben poder elegir usar o no un ILD. Sin embargo, parece que los psiquiatras suelen asumir que los pacientes no van a aceptar estos ILD y siguen empleando fármacos orales de manera mayoritaria. 
Nuestra experiencia nos muestra que sin duda habrá pacientes que lo encuentran coercitivo o estigmatizante, o simplemente no deseen una modalidad inyectable, mientras que habrá otros pacientes que lo encuentren preferible al oral por comodidad y por valorar toda una serie de ventajas que pueda haberles expuesto el clínico.

El hecho de que tradicionalmente los psiquiatras hayan centrado su prescripción de ILD en aquellos pacientes con un curso evolutivo crónico, con historia de pobre tolerancia oral y recaídas, o pacientes con gran alteración conductual y agresividad no es un hecho menor. Cabe preguntarse si de algún modo este perfil de prescripción no está de hecho favoreciendo una visión negativa y estigmatizante del uso de ILD.

Supongamos que el contexto fuera diferente, y que las pautas de prescripción habituales fueran focalizar el uso de ILD en pacientes jóvenes, con una enorme funcionalidad potencial a conservar, con el objetivo de mantener a estos pacientes libres de recaídas, hospitalizaciones, y dentro de un plan terapéutico individualizado global que persiga la normalidad en el funcionamiento psicosocial.

En esta situación hipotética, quizá alguna de las creencias o estereotipos asociados a los ILD no tuviera tanto respaldo, y se habría generado un clima favorable que no actuaría de barrera para que un nuevo paciente, en caso de que se decidiera claramente la indicación de un ILD, lo rechazara en base a estos prejuicios.

Tampoco es nuestra función negar lo evidente. Varios estudios como el de Potkin et al. recogen las actitudes de los pacientes a los ILD y muestran que es frecuente la visión negativa hacia estos fármacos o la percepción de que puedan ser más coercitivos que los antipsicóticos orales.

Potkin, S., Bera, R., Zubek, D. and Lau, G. (2013)

Patient and prescriber perspectives on long-acting

injectable (LAI) antipsychotics and analysis of

in-office discussion regarding LAI treatment

for schizophrenia. BMC Psychiatry 13: article

261 .

Sin embargo, también es una realidad que hasta en un $50 \%$ de los pacientes que reciben antipsicóticos AP orales no han recibido información acerca de las características de los ILD, las ventajas y desventajas potenciales que tienen, ni se les ha comentado la posibilidad real de que un ILD puede ser en su caso una alternativa terapéutica.

¿Qué tipo de respuestas podemos encontrar en los pacientes cuando se les ofrece ILD por primera vez?

A pesar de que los psiquiatras suelen presuponer un elevado porcentaje de rechazo hacia el mismo, parece que en realidad coexiste todo un abanico de reacciones. Caroli et al. recogieron en su muestra reacciones de tipo neutral (47\%), reacciones de tipo favorable (16\%) y reacciones desfavorables (37\%), de modo que las reacciones claramente negativas pueden no ser ni siquiera las predominantes.

Caroli, F., Raymondet, P., Izard, I., Plas, J., Gall, B. and Delgado, A. (2011) Opinions of French patients with schizophrenia regarding injectable medication. Patient Prefer Adherence 5: 165-171.

Aún más, existe un subgrupo de pacientes que, una vez cuentan con la experiencia de ambos tipos de abordaje, prefieren el ILD al oral y ofrecen argumentos para ello como por ejemplo el que se «sienten mejor», que tienen una vida más normal o que les facilita el tratamiento. 
Por estos motivos, finalmente no hay que olvidar que más allá de la subjetividad, prejuicios u opiniones, la evidencia científica nos alerta que la pobre adherencia al tratamiento oral es una de las causas principales de recaída en los casos de primer episodio psicótico.

\section{Emsley, R., Chiliza, B., Asmal, L., Mashile, M. and Fusar-Poli, P. (2013) Long-acting injectable antipsychotics in early psychosis: a literature review. Early Interv Psychiatry 7: 247-254.}

El impacto de este fenómeno no es nada despreciable: hasta un $70 \%$ de estos pacientes recaen en el primer año de evolución. Animamos al lector a la excelente revisión de Emsley et al. acerca del papel de los ILD en las primeras fases de la enfermedad esquizofrénica.

Este hecho cobra aún más importancia al tener en cuenta el fenómeno "ventana" de 5 años tras el primer episodio psicótico: el mayor deterioro clínico y psicosocial, empeoramiento cognitivo y cambios estructurales en el volumen cerebral ocurren dentro de los primeros 5 años tras el inicio de la enfermedad. El valor de evitar una segunda o tercera recaída tras el diagnóstico de esquizofrenia puede ser mucho mayor que el de evitar una decimoquinta recaída en el curso crónico recidivante avanzado del proceso. Quizás debamos maximizar todos los esfuerzos y utilizar todas las herramientas disponibles para cambiar el curso de la enfermedad en esos primeros 5 años.

¿Y si los ILD pudieran ofrecer beneficios adicionales a los antipsicóticos orales en los pacientes con un primer episodio psicótico?

Es posible que los ILD tengan su utilidad en un momento crucial del proceso donde el conocimiento sobre la enfermedad es bajo y la ambivalencia hacia la medicación es alta.

\footnotetext{
BMIJ. 2006 Jul 29;333(7561):224. Epub 2006 Jul 6
}

Effectiveness of antipsychotic treatments in a nationwide cohort of patients in community care after first hospitalisation due to schizophrenia and schizoaffective disorder: observational follow-up study.

\section{Tiihonen J ${ }^{1}$, Wahlbeck K, Lönnqvist J, Klaukka T, loannidis JP, Volavka J, Haukka J.}

- Estudio en Finlandia a gran escala, naturalístico, con más de 2000 participantes.

- Los pacientes con un primer episodio psicótico a tratamiento con ILD tenían una probabilidad 59\% menor de abandonar el tratamiento en un plazo de hasta en 7 años de seguimiento así como una probabilidad 36\% menor de reingreso en el plazo de 2 años

Una postura que puede considerarse pertinente sería no negar la opción de utilizar los ILD en los pacientes con primer episodio psicótico, dentro de un enfoque de toma de decisiones compartidas entre paciente y clínico, intentando elegir siempre la mínima dosis eficaz y eligiendo el ILD que ofrezca a cada paciente un menor perfil de efectos secundarios. 


\section{Cómo inTRoducir EL ILD}

Una vez que hemos consensuado con el paciente la introducción de un tratamiento ILD y seleccionado el mismo en relación con las premisas previamente expresadas (preferencia del paciente, antecedentes de respuesta previa a antipsicóticos, el estado global de salud y el perfil de efectos adversos) nos podremos encontrar con tres diferentes puntos de partida:

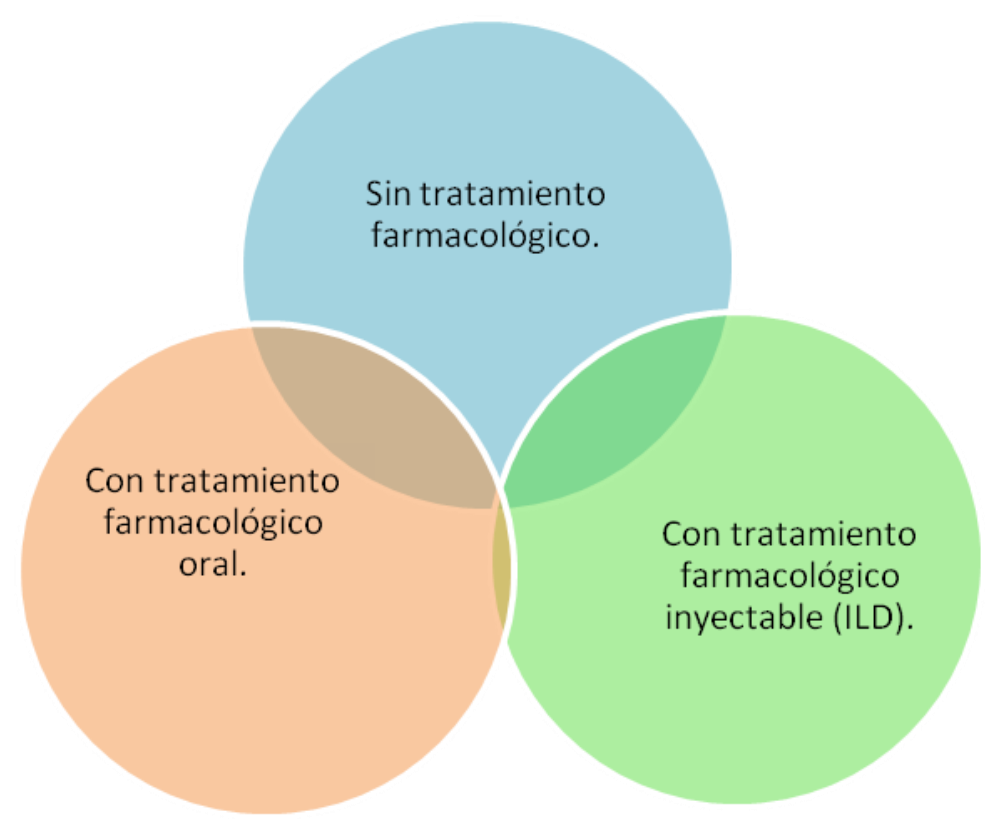

Gráfico 2: Tratamientos farmacológicos.

En el primer escenario en el cual nos planteamos introducir un fármaco ILD en un paciente de novo, es importante una correcta evaluación inicial del perfil del fármaco. Dependiendo del mismo, algunos precisan una primera fase oral que permite una mayor velocidad de ascenso de niveles en plasma que por otra parte aporta un periodo de ventana ("periodo de observación") en el que se puede objetivar sintomatología adversa subsidiaria de una suspensión y posterior cambio de antipsicótico. Dentro de estos fármacos podemos mencionar por ejemplo al aripiprazol y su periodo ventana previo a la administración del ILD.

En contrapartida los fármacos como la paliperidona, la olanzapina y la risperidona en sus formulaciones ILD no exigen como requisito la administración oral previa aunque siempre es recomendable para ensayar tolerancia.

Además de estos fármacos del grupo de los APSG, los fármacos del grupo de los APPG tampoco exigen la fase de administración oral previa a pesar de presentar un perfil de tolerabilidad/efectos adversos más complejos que los APSG. De nuevo, es recomendable ensayar tolerancia previa.

Es por esto que consideramos importante la consideración por parte del clínico de la introducción en su práctica clínica habitual del periodo de ventana oral teniendo en cuenta los beneficios que aportaría al paciente debido a que entre las principales causas de abandono del tratamiento farmacológico se sitúan los efectos adversos asociados al tratamiento antipsicótico.

No debemos perder de vista que el objetivo final es la recuperación integral del paciente pero así tan bien la prevención de recaídas como de recidivas hechos que están en estrecha relación con una adecuada adherencia al tratamiento. 
En el segundo escenario, con tratamiento oral previo, deberíamos tener en cuenta algunas de las consideraciones enunciadas previamente aunque ya tenemos antecedentes de repuesta a tratamiento con antipsicóticos: el paciente se encuentra en tratamiento oral por lo que plantear un cambio inicial a otro fármaco oral facilita el periodo ventana y la aceptación por parte del paciente; se debe utilizar la adecuada tolerancia al mismo durante ese periodo como punta de lanza para enfatizar la administración del mismo fármaco pero en formulación depot.

Y por último en el tercer escenario, en el cual plantearíamos un cambio de ILD por otro teniendo en cuenta dos aspectos: 1) la causa subyacentes que motivan el cambio (mala tolerancia, respuesta ineficaz, etc) y 2) el contexto en el cual se desarrolla (ejemplo: paciente ambulatorio vs ingresado en unidad de hospitalización). Estos dos aspectos se tendrán en cuenta a la hora de seleccionar la estrategia más adecuada de cambio así como el fármaco a elegir debido a que en esta situación deberemos tener en cuenta el perfil del fármaco previo:

- Si éste presenta propiedades antihistaminérgicas o anticolinérgicas elevadas (Olanzapina, Flufenazina o Perfenazina en ILD), existe la posibilidad de estar en presencia de receptores sensibilizados o sobre-expresados que requieran una disminución de dosis más lenta para evitar el llamado "rebote" (agitación, insomnio, ansiedad, sedación, SEP y acatisia).

A continuación se exponen los diferentes tipos de estrategias que se pueden implementar en la práctica clínica diaria

\section{Tipos de estrategias de cambio de antipsicóticos:}

- Cambio brusco: interrupción inmediata del antipsicótico previo e inicio a las dosis habituales del nuevo antipsicótico.

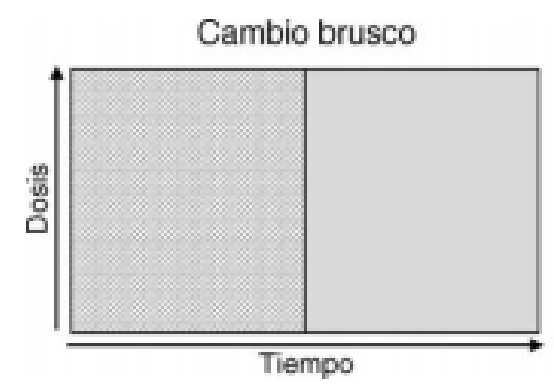

- Escalonamiento cruzado: al mismo tiempo que se procede a la retirada gradual del antipsicótico previo se inicia el incremento gradual de la dosis del nuevo antipsicótico.

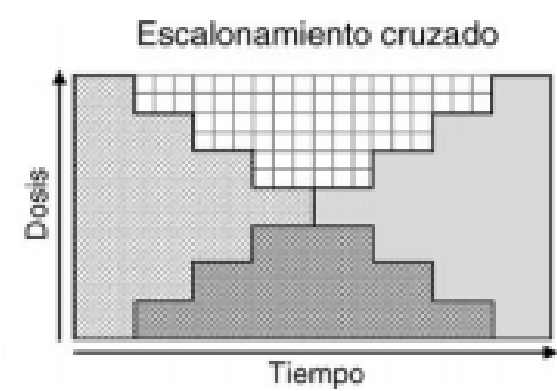


- Solapamiento y retirada: mantenimiento de las dosis del antipsicótico previo y concomitantemente inicio gradual del nuevo antipsicótico hasta alcanzar dosis eficaces; una vez alcanzadas dosis eficaces del nuevo antipsicótico, se procede a la retirada gradual del previo.

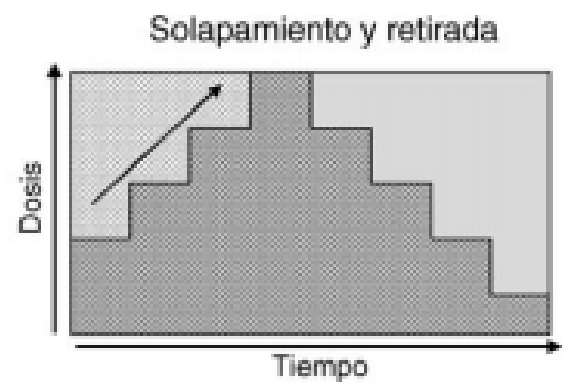

Ventajas e inconvenientes de los distintos tipos de estrategia de cambio:

\begin{tabular}{|c|c|c|}
\hline $\begin{array}{l}\text { TIPO DE } \\
\text { CAMBIO }\end{array}$ & VENTAJAS & INCONVENIENTES \\
\hline BRUSCO & $\begin{array}{c}\text { Rápido } \\
\text { Sencillo } \\
\text { Menos errores de medicación }\end{array}$ & $\begin{array}{l}\text { Mayor riesgo de exacerbación de } \\
\text { síntomas } \\
\text { Mayor riesgo de reacciones de retirada } \\
\text { Inadecuado para cambio desde } \\
\text { Clozapina } \\
\text { Requiere supervisión más estrecha }\end{array}$ \\
\hline CRUZADO & $\begin{array}{c}\text { Puede aliviar SEP } \\
\text { Menos reacciones de retirada } \\
\text { Menos riesgo de recaídas } \\
\text { Menos riesgo de insomnio y } \\
\text { agitación } \\
\text { Mayor comodidad para el médico }\end{array}$ & Posibilidad de dosis infra-terapéuticas \\
\hline $\begin{array}{c}\text { SOLAPAMIENTO } \\
\text { Y RETIRADA }\end{array}$ & $\begin{array}{l}\text { Menos reacciones de retirada } \\
\text { Menor riesgo de recaídas } \\
\text { Menor riesgo de insomnio y } \\
\text { agitación } \\
\text { Apropiado para pacientes } \\
\text { recientemente estabilizados }\end{array}$ & $\begin{array}{l}\text { Mayor riesgo de polifarmacia } \\
\text { Mayor riesgo de reacciones adversas } \\
\text { Mayor riesgo de interacciones }\end{array}$ \\
\hline
\end{tabular}

Tabla 4: Ventajas e inconvenientes en las distintas estrategias de cambio.

La elección de uno u otro tipo de estrategia de cambio deberá hacerse adaptándose a cada caso y dependerá, entre otros, de los aspectos farmacológicos ya mencionados, de los motivos concretos del cambio (por ejemplo, la aparición de un efecto adverso grave como una agranulocitosis requiere la interrupción rápida del antipsicótico) y del marco terapéutico (por ejemplo, es más factible un cambio brusco de antipsicóticos en pacientes ingresados que aquellos en régimen ambulatorio). 


\section{Papel De la entrevista motivacional}

Parte de la pericia exigible a los clínicos es el conocimiento en profundidad de la entrevista motivacional. Dentro del contexto de una toma de decisiones compartida, abordaremos aquí brevemente algunas pinceladas de las posibles rutas que puede adoptar una entrevista con el paciente donde se aborde la opción de utilizar un ILD.

Presentamos el siguiente algoritmo donde se expresa por medio de un flujograma los distintos itinerarios que puede adoptar la entrevista clínica centrada en la propuesta de un ILD como terapia farmacológica a un paciente que se valora pueda ser un candidato que se beneficie del mismo.

Recogemos la actitud del clínico hacia el ILD, los enfoques de introducción del tema en la entrevista, las posibles respuestas del paciente, la actitud del clínico ante la reacción del paciente, y el resultado final de la entrevista.

Rogamos al lector preste especialmente atención a las fechas de flujo desde los distintos conceptos reflejados: una vía abre varias opciones las cuales pueden ser comunes a otra vía diferente. En todo caso, las rutas situadas más hacia la izquierda del flujograma orientan hacia rutas terapéuticas ideales durante la entrevista; las rutas situadas más hacia la derecha se aproximan sin embargo al ámbito de creación de barreras a la implantación del ILD.

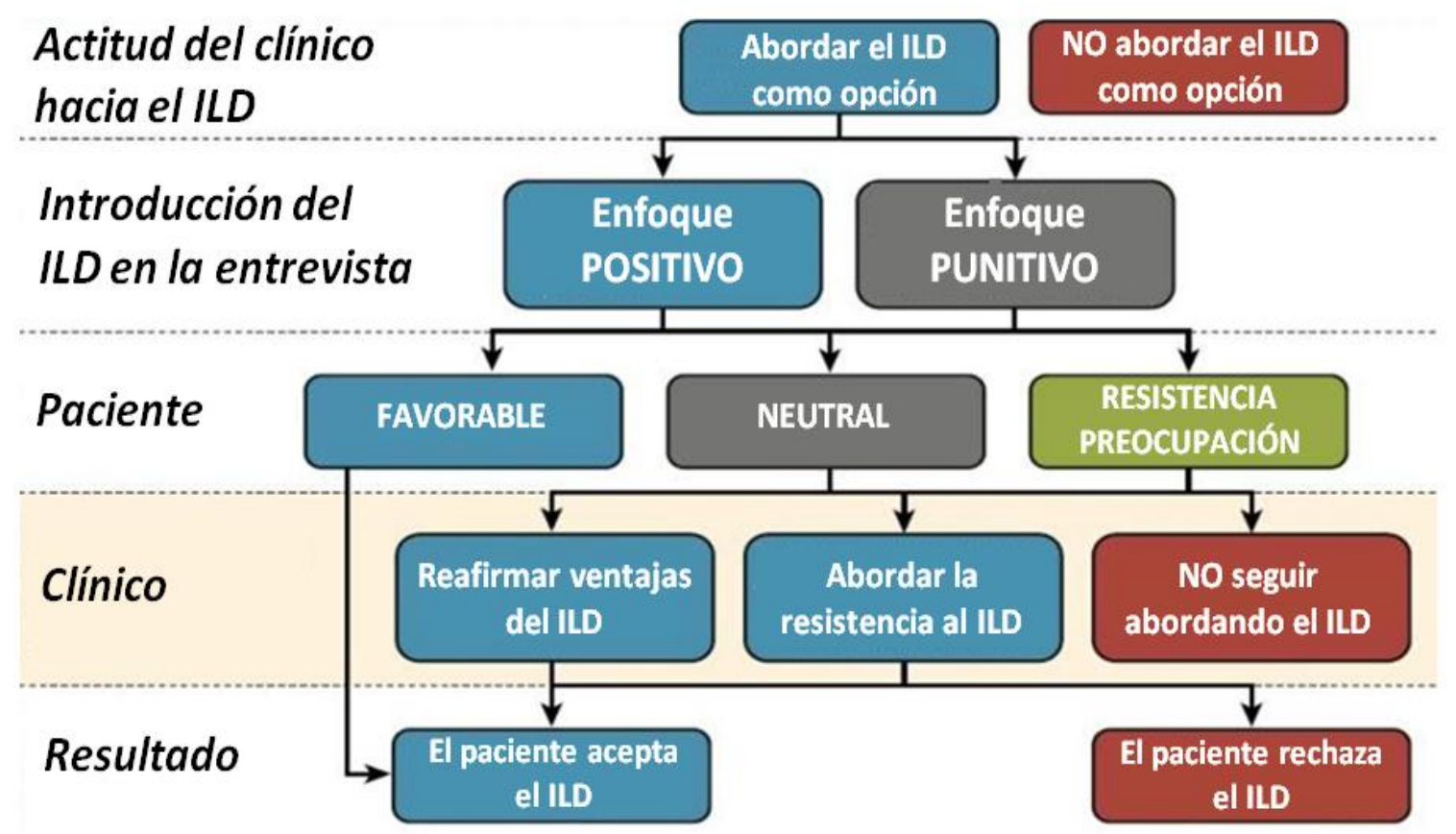

Figura 6: Rutas terapéuticas.

\section{Respecto a la actitud del clínico hacia el ILD:}

- Si ni siquiera se aborda la opción del ILD en la entrevista, es evidente que no hay margen alguno para trabajar con el paciente. Para poder explorar las posibles respuestas del paciente a la propuesta de un antipsicótico en su modalidad de larga duración, el clínico debe al menos presentar dicha opción en el transcurso de la entrevista. 
- El clínico que, valorando las ventajas y desventajas que un ILD aportarían al caso particular que tiene en consulta, decide abordar la opción del inyectable, puede hacerlo -resumido de manera necesariamente simplista- desde dos tipos de enfoques.

\section{Introducción del ILD en la entrevista:}

- Desde un enfoque positivo: el clínico le explicará al paciente las ventajas adicionales que un ILD presenta frente a un antipsicótico oral. Así mismo, puede incidir en historias de éxito que otros pacientes antes que él han tenido con el ILD. Desde este enfoque, no hay lugar para amenazas, coacciones, o señalar (directa o indirectamente) que el ILD viene a solucionar una pobre o nula adherencia terapéutica del paciente.

- Desde un enfoque punitivo: este enfoque no se centra en resaltar aquello de positivo o ventajoso que puede ofrecer el ILD al paciente, sino en los problemas que el paciente presenta y el ILD acude a paliar. Explicar que el ILD viene a solucionar su falta de compromiso con el tratamiento antipsicótico oral, exponiendo de manera más o menos velada estrategias coercitivas o basadas en el miedo, pueden de hecho acabar logrando que el paciente acepte el ILD, aunque debemos preguntarnos si realmente queremos que la conversación durante la entrevista se dirija en estos términos y su justificación final se base en este tipo de argumentos.

\section{Respuesta del paciente al enfoque elegido por el clínico:}

- Desde un enfoque positivo: se pueden obtener respuestas favorables a la incorporación del ILD como opción, respuestas neutrales (mostrando ambivalencia por ambas opciones y no una inclinación clara por las ventajas o desventajas del mismo) y respuestas desfavorables (básicamente de resistencia a la nueva opción o de preocupación por aspectos concretos de la misma). Aunque los tres tipos de respuesta son posibles, terapéuticamente este enfoque parece predisponer al paciente hacia respuestas más favorables.

- Desde un enfoque punitivo: se pueden obtener así mismo respuestas favorables a la incorporación del ILD como opción (puede haber funcionado la estrategia de amenaza latente, coerción, preferencia por evitar un ingreso hospitalario, culpabilidad por no haber respetado el régimen terapéutico oral...), respuestas neutrales (mostrando ambivalencia por ambas opciones y no una inclinación clara por las ventajas o desventajas del mismo) y respuestas desfavorables (siendo desde este enfoque punitivo más frecuente encontrar respuestas de resistencia ante, por ejemplo, la imposición velada de la nueva opción desde una posición de autoridad y poder por parte del clínico o respuestas de preocupación por aspectos concretos de la misma como la pérdida de autonomía, la pérdida de capacidad de control sobre el tratamiento...). Aunque los tres tipos de respuesta son posibles, terapéuticamente este último enfoque parece predisponer al paciente hacia respuestas más desfavorables. 


\section{$\underline{\text { Respuesta del clínico ante la reacción del paciente: }}$}

- Si la respuesta del paciente ha sido favorable al enfoque elegido por el clínico: el clínico pasará directamente a instaurar el ILD dentro de un plan terapéutico individualizado.

- Si la respuesta del paciente ha sido neutral al enfoque elegido por el clínico: el clínico deberá decidir básicamente entre 3 tipos de estrategias $\rightarrow$ puede decidir entre enfatizar las ventajas del ILD, resolviendo dudas y aclarando hasta donde sea preciso; puede optar por analizar en detalle los componentes de resistencia y preocupación del paciente para poder aflorar explícitamente en la entrevista el grado de intensidad de la resistencia, sus componentes concretos y superar las posibles objeciones que tenga el paciente desde la argumentación, comprensión y respeto; una última opción ante una respuesta neutral del paciente también podría ser no insistir más en la opción del ILD y abandonar la discusión de este aspecto en la entrevista. Esta última opción cierra el abordaje del ILD.

- Si la respuesta del paciente ha sido desfavorable al enfoque elegido por el clínico: el clínico deberá decidir también entre los mencionados 3 tipos de estrategias $\rightarrow$ puede decidir entre enfatizar las ventajas del ILD, resolviendo dudas y aclarando hasta donde sea preciso con la esperanza de que reforzando los aspectos positivos del ILD el paciente pueda modular su respuesta; la segunda estrategia cobra aquí mayor relevancia, y es mandatorio analizar en detalle los componentes de resistencia y preocupación del paciente para poder aflorar explícitamente en la entrevista el grado de intensidad de la resistencia, sus componentes concretos y superar las posibles objeciones que tenga desde la argumentación, comprensión y respeto; la última opción de no insistir más en la oferta del ILD es más probable ante una respuesta desfavorable; sin embargo, antes de tomar este camino, debemos preguntarnos si abandonar la discusión de este aspecto en la entrevista cierra una alternativa útil a un paciente que quizás pueda beneficiarse más incluso que otros casos. Decidir seguir trabajando la opción del ILD cuando enfrentamos una negativa clara exige un esfuerzo extraordinario por parte del clínico y unos sólidos fundamentos y pericia en el manejo de la entrevista motivacional.

\section{Resultado final de la entrevista:}

- El paciente acepta el ILD como opción: si el paciente acepta recibir el ILD este resultado puede haberse alcanzado a través de distintos tipos de enfoques, a partir de distintas respuestas del paciente, y a partir de distintas reacciones del clínico. Sin embargo, queda claro que hay una vía por la que nunca se podrá llegar a este resultado final: no abordar la opción del ILD en la entrevista.

- El paciente rechaza el ILD como opción: de manera análoga, la negativa del paciente al ILD es un resultado al que llegar a través de distintos tipos de enfoques, distintas respuestas del paciente y distintas reacciones del clínico. Los enfoques punitivos no son los óptimos; el esfuerzo y tiempo necesario 
para resolver estas barreras pueden hacer al clínico desistir finalmente en la estrategia.

Obviamente, el clínico debe conocer los fundamentos teóricos de la entrevista motivacional y contar con suficiente experiencia en esta técnica como para poder resolver con soltura las difíciles situaciones a las que seguro va a enfrentarse. En el gráfico que ofrecemos a continuación se pretenden reflejar una serie de estilos de entrevista con sus componentes relativos:

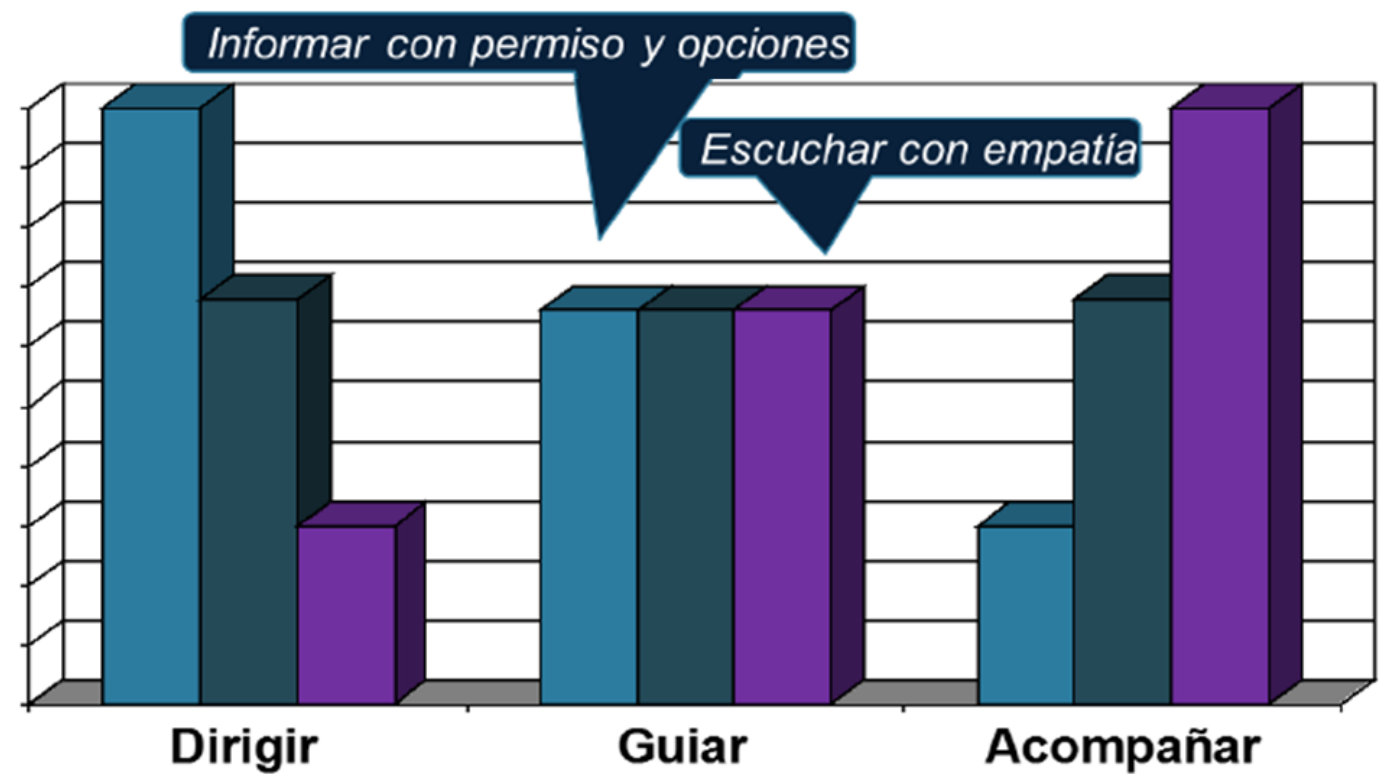

\section{$\square$ Informar $\square$ Preguntar $\square$ Escuchar}

Gráfico 3: Estilos de entrevista con sus componentes relativos.

De manera resumida, puede decirse que existen 3 estilos diferentes de dirigir la entrevista, basados en el tipo de distribución e importancia relativa que se le otorga a 3 herramientas:

- Dirigir: el clínico puede sentirse más cómodo dirigiendo la entrevista, y para ello dará preponderancia a ofrecer información, además de utilizar también preguntas. El foco de este estilo directivo ofrece una menor importancia relativa a la escucha.

- Guiar: en este estilo, el clínico estructura su entrevista repartiendo de una manera similar las actitudes de solicitar información, formular preguntas y escuchar al paciente, equilibrando todas estas técnicas.

- Acompañar: finalmente, el clínico puede decidir que lo más importante de la entrevista es la escucha empática al paciente, realizando preguntas para aclarar o reenfocar el tema, y dedicando una menor importancia relativa a la posición de informador experto. 
Muchas veces se plantea la duda acerca cuál es el mejor estilo para utilizar en los pacientes con un trastorno mental grave. La respuesta, a nuestro juicio, es siempre la misma:

No existe un estilo óptimo de entrevista que se adecue a todos los pacientes de manera universal

Serán las características específicas de cada caso concreto las que deben orientarnos sobre el estilo que mejor resultado va a ofrecer en la entrevista

La conclusión, pues, es obvia: el clínico debe contar con un nivel de pericia suficiente para moverse entre todos los estilos con naturalidad y comodidad para poder adaptar su comunicación a la que el paciente pueda requerir en cada situación. 


\section{Bibliografía}

Parellada E, Bioque M. Barriers to the Use of Long-Acting Injectable Antipsychotics in the Management of Schizophrenia. CNS Drugs. 2016 Jun 2;PubMed PMID: 27255405.

Singh SM, Haddad PM, Husain N, Heaney E, Tomenson B, et al. Cross-sectional comparison of firstgeneration antipsychotic long-acting injections vs risperidone long-acting injection: patientrated attitudes, satisfaction and tolerability. Ther Adv Psychopharmacol. 2016 Jun;6(3):16271. PubMed PMID: 27354904; PubMed Central PMCID: PMC4910399.

Ertem M, Duman ZÇ. Motivational Interviewing in a Patient With Schizophrenia to Achieve Treatment Collaboration: A Case Study. Arch Psychiatr Nurs. 2016 Apr;30(2):150-4. PubMed PMID: 26992863.

Montemagni C, Frieri T, Rocca P. Second-generation long-acting injectable antipsychotics in schizophrenia: patient functioning and quality of life. Neuropsychiatr Dis Treat. 2016;12:91729. PubMed PMID: 27143893; PubMed Central PMCID: PMC4844443.

Baandrup L, Østrup Rasmussen J, Klokker L, Austin S, Bjørnshave T, et al. Treatment of adult patients with schizophrenia and complex mental health needs - A national clinical guideline. Nord J Psychiatry. 2016;70(3):231-40. PubMed PMID: 26328910.

Chien WT, Mui JH, Cheung EF, Gray R. Effects of motivational interviewing-based adherence therapy for schizophrenia spectrum disorders: a randomized controlled trial. Trials. 2015 Jun 14;16:270. PubMed PMID: 26072311; PubMed Central PMCID: PMC4469254.

Vanderwaal FM. Impact of Motivational Interviewing on Medication Adherence in Schizophrenia. Issues Ment Health Nurs. 2015;36(11):900-4. PubMed PMID: 26631862.

Majer IM, Gaughran F, Sapin C, Beillat M, Treur M. Efficacy, tolerability, and safety of aripiprazole once-monthly versus other long-acting injectable antipsychotic therapies in the maintenance treatment of schizophrenia: a mixed treatment comparison of double-blind randomized clinical trials. J Mark Access Health Policy. 2015;3PubMed PMID: 27123183; PubMed Central PMCID: PMC4802697.

McEvoy JP, Byerly M, Hamer RM, Dominik R, Swartz MS, et al. Effectiveness of paliperidone palmitate vs haloperidol decanoate for maintenance treatment of schizophrenia: a randomized clinical trial. JAMA. 2014 May 21;311(19):1978-87. PubMed PMID: 24846035; NIHMSID: NIHMS602159; PubMed Central PMCID: PMC4101890.

Rosenheck R, Lin H. Noninferiority of perphenazine vs three second-generation antipsychotics in chronic schizophrenia. J Nerv Ment Dis. 2014 Jan;202(1):18-24. PubMed PMID: 24375207; NIHMSID: NIHMS731368; PubMed Central PMCID: PMC4635461. 
Potkin S, Bera R, Zubek D, Lau G. Patient and prescriber perspectives on long-acting injectable (LAI) antipsychotics and analysis of in-office discussion regarding LAl treatment for schizophrenia. BMC Psychiatry. 2013 Oct 16;13:261. PubMed PMID: 24131801; PubMed Central PMCID: PMC3819472.

Emsley R, Chiliza B, Asmal L, Mashile M, Fusar-Poli P. Long-acting injectable antipsychotics in early psychosis: a literature review. Early Interv Psychiatry. 2013 Aug;7(3):247-54. PubMed PMID: 23342964.

Lammers L, Zehm B, Williams R. Risperidone long-acting injection in Schizophrenia Spectrum Illnesses compared to first generation depot antipsychotics in an outpatient setting in Canada. BMC Psychiatry. 2013 May 30;13:155. PubMed PMID: 23718192; PubMed Central PMCID: PMC3669627.

Geerts P, Martinez G, Schreiner A. Attitudes towards the administration of long-acting antipsychotics: a survey of physicians and nurses. BMC Psychiatry. 2013 Feb 17;13:58. PubMed PMID: 23414331; PubMed Central PMCID: PMC3599671.

Covell NH, McEvoy JP, Schooler NR, Stroup TS, Jackson CT, et al. Effectiveness of switching from long-acting injectable fluphenazine or haloperidol decanoate to long-acting injectable risperidone microspheres: an open-label, randomized controlled trial. J Clin Psychiatry. 2012 May;73(5):669-75. PubMed PMID: 22480442; NIHMSID: NIHMS494877; PubMed Central PMCID: PMC3739692.

Caroli F, Raymondet P, Izard I, Plas J, Gall B, et al. Opinions of French patients with schizophrenia regarding injectable medication. Patient Prefer Adherence. 2011 Mar 21;5:165-71. PubMed PMID: 21573047; PubMed Central PMCID: PMC3090377.

Leucht S, Corves C, Arbter D, Engel RR, Li C, et al. Second-generation versus first-generation antipsychotic drugs for schizophrenia: a meta-analysis. Lancet. 2009 Jan 3;373(9657):31-41. PubMed PMID: 19058842.

Jones PB, Barnes TR, Davies L, Dunn G, Lloyd H, et al. Randomized controlled trial of the effect on Quality of Life of second- vs first-generation antipsychotic drugs in schizophrenia: Cost Utility of the Latest Antipsychotic Drugs in Schizophrenia Study (CUtLASS 1). Arch Gen Psychiatry. 2006 Oct;63(10):1079-87. PubMed PMID: 17015810.

Tiihonen J, Wahlbeck K, Lönnqvist J, Klaukka T, loannidis JP, et al. Effectiveness of antipsychotic treatments in a nationwide cohort of patients in community care after first hospitalisation due to schizophrenia and schizoaffective disorder: observational follow-up study. BMJ. 2006 Jul 29;333(7561):224. PubMed PMID: 16825203; PubMed Central PMCID: PMC1523484.

Rubio G, Martínez I, Ponce G, Jiménez-Arriero MA, López-Muñoz F, et al. Long-acting injectable risperidone compared with zuclopenthixol in the treatment of schizophrenia with substance abuse comorbidity. Can J Psychiatry. 2006 Jul;51(8):531-9. PubMed PMID: 16933590. 
Heres S, Davis J, Maino K, Jetzinger E, Kissling W, et al. Why olanzapine beats risperidone, risperidone beats quetiapine, and quetiapine beats olanzapine: an exploratory analysis of head-to-head comparison studies of second-generation antipsychotics. Am J Psychiatry. 2006 Feb;163(2):185-94. PubMed PMID: 16449469.

Goff DC, Sullivan LM, McEvoy JP, Meyer JM, Nasrallah HA, et al. A comparison of ten-year cardiac risk estimates in schizophrenia patients from the CATIE study and matched controls. Schizophr Res. 2005 Dec 1;80(1):45-53. PubMed PMID: 16198088.

Rosenheck R, Perlick D, Bingham S, Liu-Mares W, Collins J, et al. Effectiveness and cost of olanzapine and haloperidol in the treatment of schizophrenia: a randomized controlled trial. JAMA. 2003 Nov 26;290(20):2693-702. PubMed PMID: 14645311. 
Medicina 